\title{
ATHEROGENIC PARAMETERS AND CARDIAC INDICES IN PROGNOSTICATION OF DEVELOPMENT OF PRE-ECLAMPSIA AND ITS CARDIOMETABOLIC CONSEQUENCES
}

\author{
Anastasiia Aksonova \\ Department of obstetrics and gynaecology N 1 \\ National O.O. Bogomolets Medical University \\ 13 Shevchenko blvd, Kyiv, Ukraine, 03150 \\ Perinatal center \\ 9 Predslavinskaya str., Kyiv, Ukraine, 03150 \\ aks.anastasia@mail.ru \\ Iryna Ventskovskaya \\ Department of obstetrics and gynaecology N 1 \\ National O. O. Bogomolets Medical University \\ 13 Shevchenko blvd, Kyiv, Ukraine, 03150 \\ Perinatal center \\ 9 Predslavinskaya str., Kyiv, Ukraine, 03150 \\ Tatyana Yuzvenko \\ Department of prevention and treatment of diabetes mellitus \\ Ukrainian Scientific-Practical Centre of Endocrine Surgery \\ Transplantation of Endocrine Organs and Tissues Ministry of Health of Ukraine \\ 13A Klovskyy descent, Kyiv, Ukraine, 01021 \\ yuzvenko@bk.ru
}

\footnotetext{
Abstract

Aim of the work. The study of interconnection between atherogenic parameters of the blood serum and cardiac indices during pregnancy with the risk of pre-eclampsia development and prognostication of remote cardio-vascular and/or metabolic pathology among pregnant.

Materials and methods. The indices of lipid metabolism in the blood serum of 267 pregnant women were studied using diagnostic sets of reagents. The blood sampling was carried out in II (16-24 gestational weeks) trimester. The general cholesterol (GC) and triglycerides (TG) were determined by colorimetric and enzymatic method; high-density lipoproteins - HDL - by homogenous method of direct determination of HDL-cholesterol concentration in the blood serum without preliminary processing and centrifugation, low-density lipoproteins - LDL - by the direct method. VLDL concentration was calculated by Friedewald formula: $\mathrm{LDL}=\mathrm{TG} / 2,2$.

For evaluation of GC-VLDL concentration TG/2,2 parameter is used, if TG concentration is expressed in mmol/1 (or TG/5, if concentration is expressed in $\mathrm{mg} / 100 \mathrm{ml}$ ).

Cardiac indices were calculated taking into account the lipid parameters according to the correspondent mathematical formulas. Lactate and LDH concentrations were determined by optimized method using diagnostic reagents. Lactate concentration was determined at the use of diagnostic set for determination of lactate concentration, and DLH was also determined by diagnostic set for determination of LDH (DL) activity by optimized method, elaborated taking into account the recommendations of German Association of clinical chemistry (DGKC) after correspondent calibration of automatic analyzer ACCENT-200 II GEN. Depending on the development of clinical presentation of PE 43 women were divided in groups with light and middle severity of course of this pathology.

Results and discussion. Among the women with PE development was observed the essential changes in II trimester in the aspect of atherogenic parameters and cardiac indices mainly at the expense of progressing increase of TG in the blood serum (mmol/1: I group - 1,73 $\pm 0,14$, II group - 1,86 $\pm 0,18$ comparing with III control group $-1,51 \pm 0,06$. The gradual growth of cardiac (atherogenic) indices - AIP, CR and CA and connection between AIP and the levels of maternal serumal TG and arterial pressure were observed in women with PE development at the absence of such connection among the women of control group $(\mathrm{p}<0,05)$. LDH level grew with the progressing of PE severity: for the women of I group LDH level was $-389 \pm 1,6 \mathrm{U} / 1$ at $778 \pm 0,25$ in women of II group and $265 \pm 0,19$ in the control group $(\mathrm{p}<0,05)$.
} 
Conclusions. It was demonstrated, that hypertriglyceridemia at pregnancy is a predicator relative to PE development and together with progressive growth of atherogenic indices can be considered as prognostic factor for cardiometabolic pathology, especially in women with undergone severe forms of this pathology. It was established, that the increase of LDH level since the second trimester can also testify to the development of PE and remote cardiac pathology in this category of women.

Keywords: pre-eclampsia, atherogenic parameters, cardiac indices, LDH.

\section{Introduction}

The pathology of cardio-vascular system remains the one of main causes of morbidity and mortality throughout the world and especially in Ukraine. For today hypertensive disorders complicate the course of $9-25 \%$ of all pregnancies and are considered as an important risk factor of CVD in women [1]. The special importance is gained by pre-eclampsia (PE) that is responsible for each third case of obstetric morbidity, for fivefold increase of perinatal mortality and minimum for 76000 maternal deaths annually throughout the world [2]. PE complicates $12-17 \%$ of pregnancies and is characterized with numerous injuries that include proteinuria, thrombocytopenia, renal failure, disorder of liver function with edema of lungs and brain, visual symptoms, but the most typical is the development of arterial hypertension (systolic arterial pressure $\geq 140$ /diastolic arterial pressure $\geq 90 \mathrm{~mm}$ of $\mathrm{m}$. c.) after the twentieth week of gestation (American college of Obstetricians and Gynecologists, 2013) [3]. It is important to note, that this pathology increases the risks of cardio-vascular pathology in future from two to four times [4]. According to the recommendations of American cardiologic association, since 2011 it is used to consider PE as independent gender-specific risk factor [5]. In this connection the search for early predicators of its development with possible elaboration of distinct prophylactic and treating arrangements on the base of these data remains topical for this time [6]. Endothelial dysfunction plays the central role in genesis of multisystem disorders that precede the development of PE clinical presentation. The mechanisms, involved in induction of endothelial cellular dysfunction are insufficiently studied for today. Today in foreign scientific community take place the researches, directed on confirmation or confutation of the possible participation of atherogenic parameters of lipid metabolism in PE pathogenesis and their important role for its early prognostication and diagnostics. At the same time the determination of several lipoprotein ratios or atherogenic (cardiac) indices [7] that can be the better alternative to the routine method of examination remain important in optimization of prognostic importance of lipid profile in genesis of remote cardio-vascular and metabolic risks after PEB. But the scientific views are contradictive and include insufficiently studied aspects and this fact, undoubtedly, indicates the actuality of further studies in this field.

\section{Aim}

The study of interconnection between atherogenic parameters of the blood serum and cardiac indices during pregnancy with the risk of pre-eclampsia development and prognostication of remote cardio-vascular and/or metabolic pathology among pregnant.

\section{Materials and methods}

According to the set aim of research, atherogenic parameters were studied in the blood serum of 267 pregnant women using diagnostic set of reagents by PZ CORMAY S. A. (Poland) after correspondent calibration of each method on automatic analyzer ACCENT-200 II GEN. The blood sampling was carried out in II (16-24 gestational weeks) trimester. Criteria of inclusion: age 19-40 years, pregnancy with one living fruit that occurred without additional reproductive technologies usage, informed consent for the research during whole period of gestation, absence of severe somatic pathology. Criteria of exclusion from the research: the high risk of PE development; many-fruit pregnancy, fetal anomalies and abnormal karyotype; ordinary miscarriage. The general cholesterol (GC) was determined by colorimetric and enzymatic method with esterase and oxidase of cholesterol (CHOD/PAP), triglycerides (TG) - by enzymatic colorimetric method using glycerophosphoric oxidase (GPO), high-density lipoproteins - HDL - were determined using the 
diagnostic set of determination of HDL cholesterol concentration by homogenous method of direct determination of HDL-cholesterol concentration in the blood serum without preliminary processing and centrifugation; low-density lipoproteins (LDL) were determined using diagnostic method for determination of LDL cholesterol concentration by the direct method. Very low-density proteins (VLDL) concentration was calculated by Friedewald formula: VLDL=TG/2,2. The calculation of atherogenic index of plasma (AIP) was carried out, taking into account the levels of lipid parameters: $\mathrm{AIP}=\mathrm{Log} \mathrm{TG} / \mathrm{HDL}-\mathrm{c}$, where TG and LHL concentration is expressed in $\mathrm{mmol} / \mathrm{l}$ [8]. The calculation of the levels of cardiac risk (CR) was carried out by the formula: $\mathrm{CR}=\mathrm{TC} / \mathrm{HDL}-\mathrm{c}$ [9]. The coefficient of atherogenicity (CA) was calculated by the following formula: $\mathrm{CA}=(\mathrm{TC}-\mathrm{HDL}-\mathrm{c}) / \mathrm{HDL}-\mathrm{c}$ [10] (referent values - 2,2-3,5). Lactate concentration was determined using diagnostic set for determination of its concentration (referent value is $0,5-2,2 \mathrm{mmol} / \mathrm{l}$ ) and $\mathrm{LDH}$ that was also determined using diagnostic set for LDH (LD) by optimized method, elaborated taking into account the recommendations of German Association of clinical chemistry (DGKC) (referent values 225-450 U/1) after correspondent calibration of automatic analyzer ACCENT-200 II GEN. Statistical processing of data was carried out using the package of applied programs Microsoft Office Excel 2010 and StatSoft Statistica 6.1 [11].

\section{Results of research}

In 43 (16,1 \%) women of 267 examined pregnancy was complicated with the development of clinical signs of PE. Among them I group included 26 women with the development of PE clinical presentation of light degree; II group - 17 women with PE of middle severity. The other 224 women formed the control group (III group) without obstetric and somatic pathology with normal course of pregnancy without PE clinic, who were delivered in time mainly by the way of natural puerperal ways (Fig. 1).

Cases of pre-eclampsia among examined women

- Women with physiological course of pregnancy $\quad$ Pre-eclampsia of light degree - Severe forms of preeclampsia

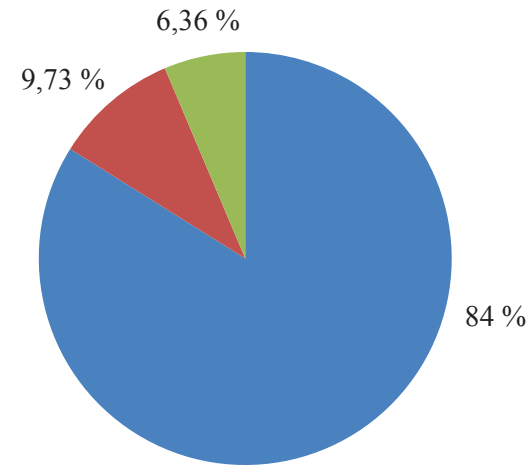

Fig. 1. Cases of pre-eclampsia (\%) among women, examined during the II trimester of pregnancy

Mean age of women (years) of I group was $31,03 \pm 0,67$, II group - 28,24 $\pm 0,68$, III group $30,04 \pm 0,54$. Body mass index (BMI) among examined groups of women was: $25,13 \pm 0,54-$ in I group, comparing with control - 21,23 $\pm 1,18$ and 27,41 $\pm 1,04$ - in II group of women.

The indices of systolic and diastolic pressure ( $\mathrm{mm}$ of $\mathrm{m}$. c.) in women with PE development reliably differed from the control group: in I group - 143,72 $\pm 0,34$ and 95,21 $\pm 1,13$ at mean values of daily proteinuria (g/day) - 0,154 $\pm 1,23$; in II group - 164,34 $\pm 1,12$ and 108,32 $\pm 0,54$ at indices $3,96 \pm 1,34 \mathrm{~g}$ /day of proteinuria comparing with III group $-112,57 \pm 1,76$ and $69,13 \pm 2,31$ at absence of protein in urine.

The percent of deliveries by the way of cesarean section was $42 \%$ in the group of women with PE.

The mean levels $(\mathrm{mmol} / \mathrm{l})$ of TG among all women with cases of PE development were - 1,79 $\pm 0,16$; HDL - 0,71 $\pm 0,12$; VLDL - 0,84 $\pm 0,08$ comparing with TG, HDL and VLDL levels among the women of control group that were respectively $1,51 \pm 0,06 ; 1,19 \pm 0,12$ and $0,60 \pm 0,16(p<0,05)$. 
These disorders of lipid metabolism were especially brightly expressed in women, whose pregnancy was complicated with the severe PE forms, that was testified by the received high indices of TG - 1,86 $\pm 0,18$ with decrease of HDL $-0,64 \pm 0,04$ and increase of VLDL $-0,90 \pm 0,06$ levels in II studied group of pregnant comparing with I main group and control group of women with physiological course of pregnancy $(\mathrm{p}<0,05)$. At analysis of GC and LDL any significant differences were not observed between the women with PE development and control group. Among all women of control group were not observed any reliable differences in all indices of lipid profile of the blood serum during II trimester of pregnancy $(\mathrm{p}<0,001)$.

In our research the mean indices of AIP in women with PE were for the I group - $0,34 \pm 0,12$, whereas for II group of women with PE of middle severity - 0,46 $\pm 0,18$ at $0,10 \pm 0,23$ in III group of physiological course of pregnancy $(\mathrm{p}<0,001)$. CR level for women with PE in I group $-6,21 \pm 0,08$ at $7,96 \pm 0,11$ in II group and 3,95 $\pm 0,19$ in control, group $(p<0,001)$. CA increase in 3,0 times was fixed among women of II group comparing with I and III groups of pregnant women (correspondingly $2,1$ and norm $)(p<0,05)$. At the same time there was demonstrated the positive connection between AIP and the levels of maternal serumal TG and systolic and diastolic arterial pressure in women with PE development at absence of such connection among women of the control group $(\mathrm{p}<0,05)$, that is testified by the results of research by Kashinakunti et al., and Bennal et al. [16, 17].

The graphic expression of the data of atherogenic (cardiac) indices in three studied groups is presented on the Fig. 2-4.

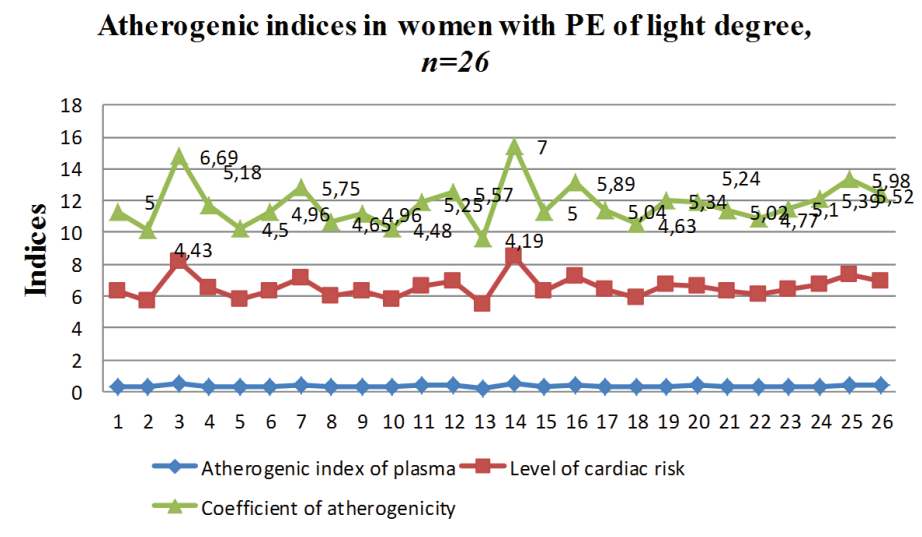

Fig. 2. Cardiac indices in women with PE of light degree (the levels of coefficient of atherogenicity among 26 women of I group are expressed in ciphers)

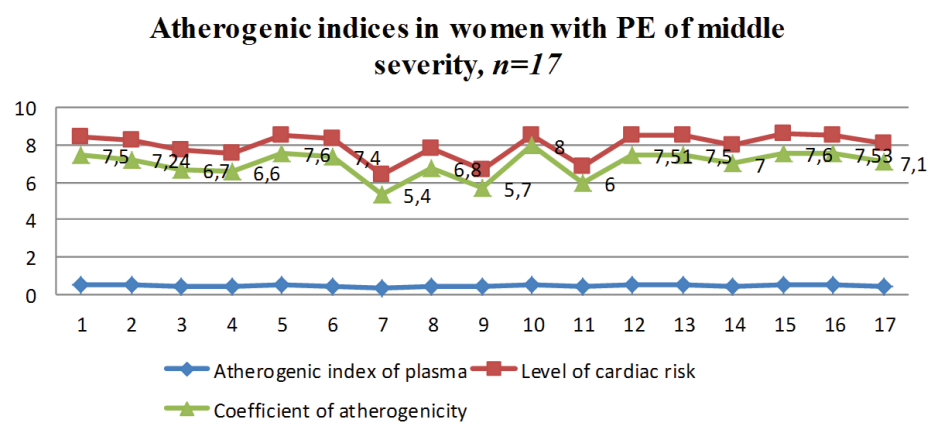

Fig. 3. Cardiac indices in women with development of middle severe forms of PE (the values of coefficient of atherogenicity among 17 women of II group are expressed in ciphers)

We also noted the gradual increase of cardiac glycolytic enzyme LDH, detected in the blood during the second trimester in women, which pregnancy was complicated with PE development, at the same time the association of this parameter especially with severe form of this pathology is important: for the women of I group LDH level was - 389 $\pm 1,6 \mathrm{U} / 1$ at $778 \pm 0,25$ in women of II group 
and $265 \pm 0,19$ in control group $(\mathrm{p}<0,05)$. At the same time the lactate level had no tendency to decrease and was within physiological norms $(\mathrm{p}<0,05)$.

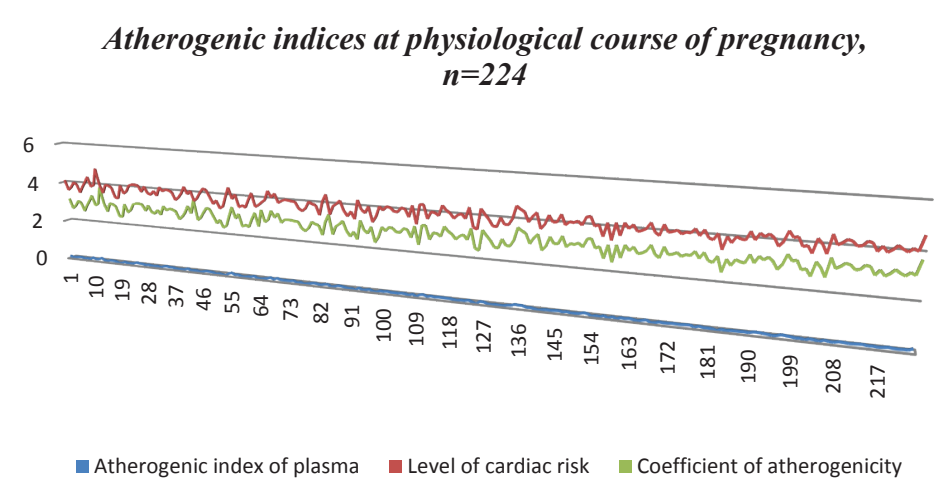

Fig. 4. Cardiac indices in women at physiological course of pregnancy

\section{Discussion of the results of research}

Analyzing the received data it is important to note, that the women of the main group with clinical signs of PE were in the young reproductive age with normal weight that is indicated by the body mass index (BMI) in studied groups.

At the analysis of indices that are responsible for the processes of initiation of atherogenesis were fixed the manifestations of dyslipoproteinemia among women with PE development mainly at the expense of hyperglyceridemia with gradual decrease of anti-atherogenic HDL and increase of atherogenic VLDL levels, fixed in the blood serum of pregnant during II gestation trimester. Taking into account the received indices of lipid profile of the blood, we calculated the modern atherogenic (cardiac) indices, which parameters can help doctors in prognostication of remote cardiometabolic pathology among this category of women with PE. Thus, according to the data of researches, AIP ("zone of arterial risk") prognosticates cardio-vascular risks and is considered as easily accessible marker of cardio-vascular pathology and is useful for determination of adequate pharmacological therapy that must not be higher than $0,24[12,13]$. According to the data of NCEP ATP III [14] the use of the level of cardiac risk (CR) can be considered as a powerful predictor of cardio-vascular risks that must not exceed 5 (optimal values of index-3,5) [15]. Analyzing the received data, we can state that in women, whose pregnancy was complicated with the development of PE clinical presentation the levels of all atherogenic indices were significantly higher.

At the same time, from our point of view, the increase of LDH level in this category can indicate the initial stages of injury of membranes of endothelial cells and increased permeability of cells for the injuring factors with activation at the beginning of compensatory possibilities of cells and their loss that is finally manifested by LDH emission in extracellular space and starting of cascade of biochemical reactions, which result is the development of endothelial dysfunction and finally clinical signs, typical for PE. Taking into account the literary sources, in women with PE development the progressive endothelial dysfunction in maternal vascular system, induced by toxic substances, released from hypoxic placenta, is a cause of deep vasoconstriction that injures practically all organs and systems, including liver. This hypoperfusion induces the ischemic injury of hepatic cells and other organs, causing the raised release of intracellular LHD in circulation channel [18-20]. At the same time the aforesaid progressive increase of LDH level in the case of PE severe forms indicates the progressing of injury of endothelium cells with the degree of severity of disease. It must be noted that in women with the development of middle severe forms of PE on the background of hypertriglyceridemia the atherogenic indices and LDH level progressively increase that can testify to the high risks of development of cardio-vascular and metabolic pathology in nearest years after delivery. This category of women is recommended to observe accurately the all prophylactic arrangements, directed on correction of hyperlipidemic states, taking into account BMI, programs of dietary nutrition and dosed physical load with obligatory biochemical control of 
lipid profile of the blood serum under the supervision of endocrinologist and cardiologist and with adequate medicamentous therapy.

\section{Conclusions}

1. Dyslipoproteinemia is a risk factor and predicator as to the development of PE and its remote cardio-vascular and metabolic complications mainly at the expense of hypertriglyceridemia, fixed in pregnant during the second gestational trimester.

2. The progressing increase of the levels of atherogenic (cardiac) indices in women with $\mathrm{PE}$, especially in the case of its middle severe forms can indicate the high risks of development of different cardio-vascular pathology in this category of women in nearest years after delivery.

3. The progressing increase of LDH level already since the second trimester of pregnancy is typical for women, whose pregnancy was complicated with the development of severe forms of PE that on the background of hypertriglyceridemic state and in correspondence with growing atherogenic indices can be the early predicator of the development of severe cardiometabolic pathology.

4. The category of women with pregnancy course, complicated with severe forms of PE is recommended to undergo the early biochemical screening as to the possible development of cardio-vascular pathology under prophylactic and medicamentous control of endocrinologist and cardiologist.

\section{References}

[1] Cunningham, F. G., Leveno, K. J., Bloom, S. L., Hauth, J. C., Rouse, D. J., Catherine, Y. S. (2010). Hypertensive disorders in pregnancy. Williams Obstetrics. New York: Mc Graw Hill Companies, 23, 706-56.

[2] Preeclampsia and maternal mortality: global burden (2013). Available at: http://www.preeclampsia.org/health-information/149-advocacy-awareness/332-preeclampsia-and-maternal-mortality-a-global-burden

[3] Hypertension in pregnancy (2013). Obstetrics \& Gynecology, 122 (5), 1122-1131. doi: 10.1097/01. aog.0000437382.03963.88

[4] Chen, C. W., Jaffe, I. Z., Karumanchi, S. A. (2014). Pre-eclampsia and cardiovascular disease. Cardiovascular Research, 101 (4), 579-586. doi: 10.1093/cvr/cvu018

[5] Mosca, L., Benjamin, E. J., Berra, K., Bezanson, J. L., Dolor, R. J., Lloyd-Jones, D. M. et. al. (2011). Effectiveness-Based Guidelines for the Prevention of Cardiovascular Disease in Women-2011 Update: A Guideline From the American Heart Association. Circulation, 123 (11), 1243-1262. doi: 10.1161/ cir.0b013e31820faaf8

[6] Mutsalhanova, Y. S. (2015). Prognostic markers of pre-eclampsia. Modern problems of science and education, 5, 326 .

[7] Bafna, A., Maheshwari, R. S., Ved, R. K., Sarkar, P. D., Batham, A. R. (2012). Study of atherogenic indices in nephrotic syndrome. International Journal of Biological and Medical Research, 3 (3), 2257-2260.

[8] Dobiasova, M., Frohlich, J., Sedova, M., Cheung, M. C., Brown, B. G. (2011). Cholesterol esterification and atherogenic index of plasma correlate with lipoprotein size and findings on coronary angiography. The Journal of Lipid Research, 52 (3), 566-571. doi: 10.1194/j1r.p011668

[9] Ikewuchi, J. C., Chigozie, J. (2010). Changes in the Weight, Plasma Lipid Profile and Atherogenic Indices of Salt-Loaded Rats by Aqueous Extract of Acalypha wilkesiana Muell Arg: Potential for Cardiovascular Risk Reduction. The Pacific Journal of Science and Technology, 11 (2), 516-523.

[10] Aryal, M., Poudel, A., Satyal, B., Gyawali, P., Pokheral, B. R., Raut, B. K. et. al. (2012). Evaluation of non-HDL-c and total cholesterol: HDL-c Ratio as Cumulative Marker of Cardiovascular Risk in Diabetes Mellitus. Kathmandu University Medical Journal, 8 (4). doi: 10.3126/kumj.v8i4.6239

[11] Rebrova, O. Y. (2006). Statistical analysis of medical data. The use of the application package STATISTICA. Moscow: Media Sphere, 305. 
[12] Nwagha, U. I., Ikekpeazu, E. J., Ejezie, F. E., Neboh, E. E., Maduka, I. C. (2010). Atherogenic index of plasma as useful predictor of cardiovascular risk among postmenopausal women in Enugu, Nigeria. African Health Sciences, 10 (3), 248-252.

[13] Emokpae, M. A., Arogundade, A., Adumanya, S. C. (2013). Use of atherogenic index of plasma in evaluating the potential cardioprotective effects of red wine consumption: Studies in Nigerian young adult volunteers. Biokemistri, 25 (3), 118-123.

[14] Third report of the national cholesterol education program (NCEP) expert panel on detection, evaluation and treatment of high blood cholesterol in adults (Adult Treatment Panel III) Final Report (2002). Circulation, 106 (25), 3143-3421. Available at: https://www.ncbi.nlm.nih.gov/pubmed/12485966

[15] Cholesterol ratio: Is it important? Mayo Clinic. Available at: http://www.mayoclinic.org/diseases.../high...ratio/faq-20058006

[16] Kashinakunti, S. V., Sunitha, H., Gurupadappa, K., Manjula, R. (2010). Lipid profile in preeclampsia - a case control study. Journal of Clinical and Diagnostic Research, 4 (4), 2748-2751.

[17] Bennal, A., Kammar, K. F. (2012). Role of Triglycerides -In Severity of Pre-Eclampsia. Journal of Physiology and Pharmacology Advances, 2 (11), 360-364.

[18] Munde, S. M., Hazari, N. R., Thorat, A. P., Gaikwad, S. B., Hatolkar, V. S. (2014). Gamma glutamyl transferase and Lactate dehydrogenase as biochemical markers of severity of preeclampsia. International Journal of Medical, Health, Biomedical, Bioengineering and Pharmaceutical Engineering, 8 (1), 50-53.

[19] Bera, S., Gupta, S., Roy, S. S., Kunti, S., Biswas, S., Ghosh, D. (2014). Study of liver enzymes especially lactate degydrogenase to predict foetal outcome in pregnancy induced hypertension. Scholars Journal of Applied Medical Sciences, 2 (5A), 1569-1572.

[20] Kozic, J. R., Benton, S. J., Hutcheon, J. A., Payne, B. A., Magee, L. A., von Dadelszen, P. (2011). Abnormal Liver Function Tests as Predictors of Adverse Maternal Outcomes in Women With Preeclampsia. Journal of Obstetrics and Gynaecology Canada, 33 (10), 995-1004. doi: 10.1016/s1701-2163(16)35048-4 